\title{
Influence of intravenous fat emulsion on serum bilirubin in very low birthweight neonates
}

\author{
Y W BRANS, D A RITTER, J D KENNY, D S ANDREW, E B DUTTON, \\ AND D W CARRILLO
}

Perinatal Research Laboratory, Departments of Pediatrics and Obstetrics and Gynecology, University of Texas Health Science Center, San Antonio, Texas, and Department of Pediatrics, Temple University School of Medicine, Philadelphia, Pennsylvania, United States of America

SUMmARY Thirty nine very low birthweight neonates (with a birth weight of 820 to $1500 \mathrm{~g}$ and gestation of 27 to 34 weeks) who required total parenteral nutrition were randomly assigned to one of three regimens of administration of fat emulsion for a period of eight days. Groups 1 and 2 received the emulsion at a constant rate over 24 and 16 hours, respectively, beginning with a daily dosage of $1 \mathrm{~g} / \mathrm{kg}$ and increasing daily by $1 \mathrm{~g} / \mathrm{kg}$ to a maximum of $4 \mathrm{~g} / \mathrm{kg}$. Group 3 received the emulsion at a constant rate of $4 \mathrm{~g} / \mathrm{kg}$ a day over 24 hours. Plasma concentrations of free fatty acids and serum concentrations of total bilirubin, apparent unbound bilirubin, and albumin were measured at regular intervals. Effects of the three regimens on serum bilirubin measurements were determined. The regimen of fat infusion and rate of infusion seemed to have no effect on serum concentrations of total and apparent unbound bilirubin, although there was a trend towards greater variability in apparent unbound concentrations with the intermittent regimen.

The use of fat emulsions for total parenteral nutrition of very low birthweight neonates has gained widespread acceptance in an attempt to provide better energy intakes. The limited metabolic tolerance of tiny neonates, shown long ago with dextrose and amino acid solutions, ${ }^{1}$ unfortunately applies also to lipids. Neonates with birth weights between 750 and $1500 \mathrm{~g}$ respond to infusion of fat emulsions with sharp increases in their plasma concentrations of total lipids, free glycerol, triglycerides, free fatty acids, and cholesterol. ${ }^{2-4}$ These increases are quite unpredictable and variable in magnitude. As the greatest need for parenteral energy occurs during the first postnatal days when the liver has not yet reached its full bilirubin conjugating potential concern has been expressed about displacement of bilirubin from albumin in the presence of high concentrations of free fatty acids. This study was designed to compare the effects of three commonly used regimens for infusion of fat emulsions on serum bilirubin measurements.

\section{Patients and methods}

Tolerance to parenterally administered fat emulsions was studied in neonates weighing $1500 \mathrm{~g}$ or less at birth. Informed parental consent was obtained in all cases. Birth weights were recorded to the nearest $10 \mathrm{~g}$. Gestational ages were determined from the mother's menstrual history, checked in most cases by sonographic determination of the biparietal diameter, and confirmed by physical examination of the neonate. ${ }^{5}$ All neonates were normally grownthat is, their birth weights were between the 10th and 90th centiles for gestational maturity, sex, and race. Separate birthweight and gestational age curves were used for hispanic (Gibbs CE. Unpublished data.), white Anglo-American, ${ }^{6}$ and black ${ }^{6}$ babies. Neonates weighing less than $750 \mathrm{~g}$ at birth or with estimated gestational ages below 27 weeks were excluded from the study.

The neonates were managed in accordance with the usual practices for our nursery. Phototherapy was used in all neonates from birth and generally continued throughout the study. Total parenteral nutrition was started on the third postnatal day unless otherwise indicated. ${ }^{4}$ The neonates received fat emulsion (Intralipid, KabiVitrum, Alameda, California, United States) according to one of three randomly allocated regimens. Those in group 1 received the fat emulsion at a constant rate over 24 hours, beginning at a daily dosage of $1 \mathrm{~g} / \mathrm{kg}$ and 
increasing by $1 \mathrm{~g} / \mathrm{kg}$ on each successive day to a daily maximum of $4 \mathrm{~g} / \mathrm{kg}$. Those in group 2 received fat emulsion at a constant rate over 16 hours followed by eight hours without infusion of fats; daily dosage was the same as in group 1 . Those in group 3 received fat emulsion at a constant rate over 24 hours with a daily dosage of $4 \mathrm{~g} / \mathrm{kg}$ from the beginning of the infusion. Total parenteral nutrition fluids, fat emulsion excepted, were administered through an arterial umbilical catheter as long as the neonate required the catheter for blood gas monitoring. If the catheter was removed the fluids were administered through peripheral veins. The fat emulsion was infused through a peripheral vein used for no other purpose. No enteral feedings were provided during the study. Amounts of blood removed for study or clinical purposes were carefully tabulated and replaced by packed red blood cells whenever $7-10 \%$ of the baby's blood volume was estimated to have been removed. The study was stopped if a baby was unable to tolerate his or her regimen of fat emulsion-that is, if the plasma was frankly creamy on inspection whenever a blood sample was obtained for the study.

The study lasted eight days. Blood samples were obtained through the umbilical catheter (after clearing the line by removing $3 \mathrm{ml}$ of blood, which were replaced after obtaining the blood sample) or by heelstick before beginning the fat emulsion and every 24 hours thereafter. Plasma concentrations of free fatty acids were determined in quadruplicate as previously described. ${ }^{2}$ Serum concentrations of total bilirubin and apparent unbound bilirubin were determined in triplicate by a direct spectrophotometric technique. ${ }^{7}$ Serum concentrations of albumin were determined in triplicate by a colorimetric technique. ${ }^{8}$ Conjugated hyperbilirubinaemia did not occur in any neonate, with all direct bilirubin values being $17 \mu \mathrm{mol} / \mathrm{l}$ or less.

Statistical analysis. Each variable was analysed by means of one way analysis of variance to detect differences between the three groups at a given time during the study. When main effect differences were detected ( $F$ test, $p \leqslant 0 \cdot 05$ ), Duncan's multiple range test was applied to locate differences between means for various groups. A significance level of $p \leqslant 0 \cdot 05$ was chosen to define significance. Within each group, the mean value for each day of study was compared with the pre-infusion value by means of a one tailed Student's paired $t$ test as mean values were predicted to increase or to remain stable but not to decrease. As eight comparisons within a set of data were made, $p \leqslant 0 \cdot 10 / 8$ or $p \leqslant 0 \cdot 013$ was needed to indicate significant differences. Relations be- tween two variables were studied by regression analysis.

\section{Results}

Of the 39 neonates who were randomly allocated to one of the study groups, one baby in group 1 was consistently unable to tolerate fat emulsion: his plasma was frankly creamy when he was receiving only $1 \mathrm{~g} / \mathrm{kg} /$ day of fat emulsion and he also poorly tolerated glucose and amino acids; his data were excluded from analysis. The remaining 38 neonates are described in the Table. Birth weights ranged from 820 to $1500 \mathrm{~g}$, gestational ages from 27 to 34 weeks, and postnatal ages at the time of enrolment from 1 to 9 days. There were no significant differences between the three groups in mean birth weights, gestational ages, and postnatal ages.

Nine babies did not complete the eight day study. In group 1 one baby died of hyaline membrane disease. In group 2 two babies required surgical treatment for necrotising enterocolitis, one died of hyaline membrane disease, one had severe hyperlipaemia, one was removed from the study at the request of the parents, and in one the lipid infusion was interrupted inadvertently. In group 3 one baby had severe hyperlipaemia and one was removed from the study when enteral feedings were started inadvertently. Data from these babies up to the time of stopping the study were included in the analysis.

Plasma concentrations of free fatty acids ranged from $0 \cdot 13$ to $7.89 \mathrm{mmol} / \mathrm{l}$. In groups 1 and 2 mean concentrations increased with the rate of lipid infusion and stabilised at a daily infusion rate of $4 \mathrm{~g} / \mathrm{kg}$. In group 3 concentrations increased sharply as soon as the lipid infusion started and remained fairly stable thereafter (Figure).

Mean serum concentrations of total bilirubin before infusion of lipids and during each day of lipid infusion are depicted in the Figure. In some in-

Table Characteristics of the 38 neonates studied. Values are No or mean (SD) [range]

\begin{tabular}{lccc}
\hline & \multicolumn{3}{l}{ Group } \\
\cline { 2 - 4 } & 1 & 2 & 3 \\
\hline No & 14 & 13 & 11 \\
Sex (M/F) & $10 / 4$ & $8 / 5$ & $4 / 7$ \\
Race (H/W/B)* & $12 / 1 / 1$ & $8 / 3 / 2$ & $8 / 3 / 0$ \\
Birth weight (g) & $1190(196)$ & $1160(218)$ & $1160(226)$ \\
& {$[820-1480]$} & {$[820-1500]$} & {$[820-1500]$} \\
Gestational age (weeks) & $29(1 \cdot 7)$ & $29(1 \cdot 3)$ & $29(1 \cdot 3)$ \\
& {$[27-34]$} & {$[27-31]$} & {$[28-31]$} \\
Postnatal age (days) & $4(1 \cdot 9)$ & $3(1 \cdot 6)$ & $3(1 \cdot 4)$ \\
& {$[1-9]$} & {$[2-7]$} & {$[2-5]$} \\
\hline
\end{tabular}

${ }^{*} \mathrm{H}$ : Hispanics; W: Anglo-American; B: Black. 
158 Brans, Ritter, Kenny, Andrew, Dutton, and Carrillo

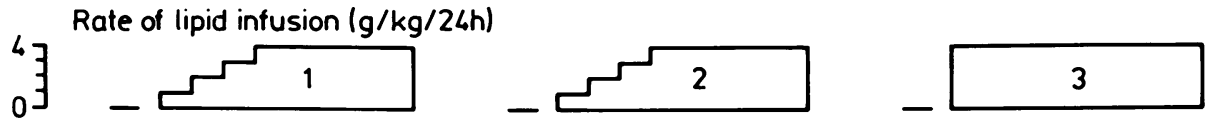

Plasma free fatty acids (mmol/I)
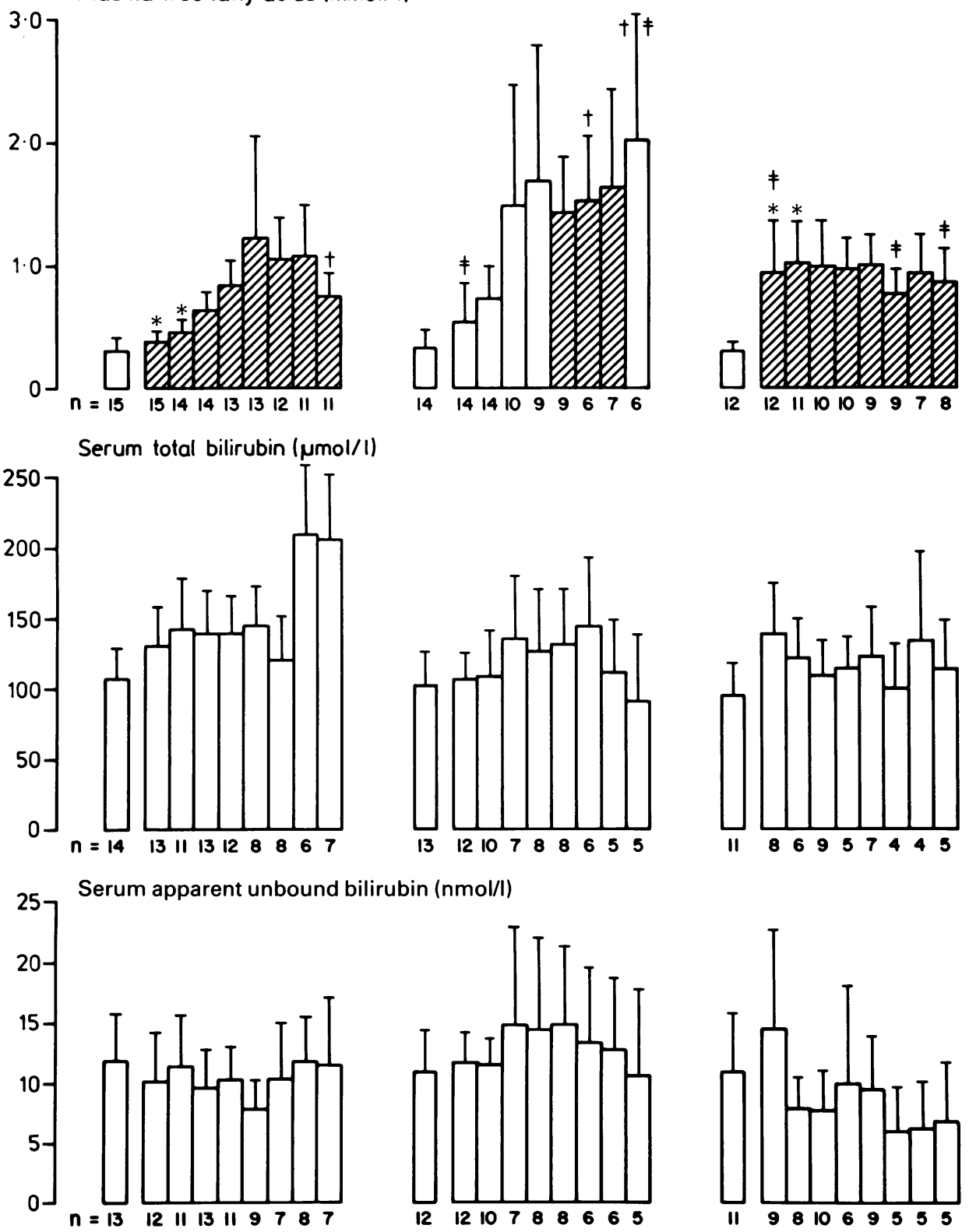

Figure Mean (2SE) plasma concentrations of free fatty acids, serum concentrations of total bilirubin, and serum concentrations of apparent unbound bilirubin before and during infusion of lipids on each of the eight days of study. The corresponding rate of lipid infusion is shown at the top. Cross hatched bars denote mean values that are significantly greater than pre-infusion values for that group ( $\mathrm{t}$ test, $p \leqslant 0.013$ ). Other symbols denote significant differences at a given time between groups 1 and $3\left({ }^{*}\right)$, groups 1 and $2(+)$ and groups 2 and $3(\ddagger)$ (one way analysis of variance and Duncan's multiple range test, $p \leqslant 0 \cdot 05)$. 
stances concentrations were not measurable due to hyperlipaemia of the sample, characterised by a visible layer of cream after the blood sample had been centrifuged. Within each group, bilirubin concentrations during infusion of lipids were not statistically different from pre-infusion concentrations-that is, the mean differences between concentrations on a given day or on a given rate of lipid intake and pre-infusion concentrations were not significantly different from zero. On a given day during the study mean bilirubin concentrations were similar for all three study groups. For individual neonates, bilirubin concentrations followed the trend that would be expected during the first one to two weeks after birth-that is, a rise followed by stable or decreasing values. No baby required any type of exchange transfusion during the study. Only five neonates in group 1, three in group 2, and three in group 3 had any measurable total bilirubin concentrations in excess of $171 \mu \mathrm{mol} / \mathrm{l}$ during the study.

Serum concentrations of apparent unbound bilirubin ranged from 1 to $45 \mathrm{nmol} / \mathrm{l}$. Only five neonates in group 1, three in group 2, and two in group $3 \mathrm{had}$ any concentrations in excess of $20 \mathrm{nmol} / \mathrm{l}$ and only one in each group had concentrations higher than $30 \mathrm{nmol} / \mathrm{l}$. There was no correlation between concentrations of apparent unbound bilirubin and concentrations of total lipids, free glycerol, triglycerides, and cholesterol. Of particular importance was the absence of correlation between concentrations of apparent unbound bilirubin and free fatty acids. Within each study group, concentrations of apparent unbound bilirubin during infusion of lipids were not statistically different from pre-infusion concentrations. On a given day of study mean concentrations were similar for all three study groups (Figure).

Serum concentrations of albumin were not affected by either duration of lipid infusion or the regimen of lipid infusion. Mean (2 SE) pre-infusion concentrations were significantly lower in group 3 than in group $1(392(21 \cdot 8) \mu \mathrm{mol} / \mathrm{l} v 441(8 \cdot 8) \mu \mathrm{mol} / \mathrm{l}$, $\mathrm{p}<0.05)$. This small difference was most likely spurious as no continuous trend was evident and the difference, though significant, was of doubtful clinical importance.

\section{Discussion}

Theoretically, parenteral fat emulsions may affect the bilirubin metabolism of a neonate in two conflicting manners.

On the one hand, particles of fat emulsions are capable of binding unconjugated bilirubin in vitro. ${ }^{910}$ This affinity for bilirubin is considerably weaker than the affinity of albumin, non-albumin serum proteins, and red blood cell membranes. ${ }^{9}$ In vitro, fat particles may displace bilirubin from red cell membranes when the bilirubin:albumin molar ratio exceeds 2 . Thus it is possible that fat particles exert some protective effect against bilirubin encephalopathy. ${ }^{10}$

On the other hand, fat emulsions are metabolised in vivo, with resulting production of free fatty acids. Although the primary binding sites of bilirubin and free fatty acids on albumin are quite distinct, free fatty acids in high concentrations may encroach on the bilirubin binding site and displace bilirubin from albumin. Liberation of bilirubin may result in a rising concentration of unbound bilirubin, potentially increasing the risk of encephalopathy. Although it is usually recommended to keep the free fatty acid:albumin molar ratio below 6 to limit the risks of toxicity, ${ }^{9}$ the ratios above which displacement of bilirubin might occur have been variably estimated at $4,{ }^{11} 125,{ }^{13} 6,{ }^{14}$ and $10 .{ }^{9}$ In fact only one study has actually shown the occurrence of free bilirubin when the ratio exceeded $6 .{ }^{14}$ Whether the circulating fat particles are able to bind the bilirubin that has been liberated by the excess of free fatty acids and to clear it through the reticuloendothelial system, thereby protecting the brain, remains speculative but is an interesting thought. There is in vitro evidence that a few of the medium chain fatty acids may improve the binding of bilirubin to albumin and that this effect varies with the length of the chain. ${ }^{15}$ In our clinical study, however, the concentration of total free fatty acids seemed to have little effect on the concentration of apparent unbound bilirubin as measured by the peroxidase assay. Free fatty acids concentrations as high as 7.89 $\mathrm{mmol} / \mathrm{l}$ were observed. Yet there was no correlation between apparent unbound bilirubin concentrations and concentrations of free fatty acids. There are at least four possible explanations for these findings:

(1) Bilirubin may not be displaced from albumin, even at these high plasma concentrations of free fatty acids.

(2) Bilirubin freed from albumin may be bound by other means-namely, non-albumin serum proteins, erythrocytic membranes, or lipid particles.

(3) Concentrations of apparent unbound bilirubin measured by the standard dilute assay may be falsely low, reflective of the 40 -fold dilution in the system.

(4) Photoisomers of bilirubin IX alpha (ZZ) may differ in their interaction with free fatty acids.

Interpretation of serum concentrations of apparent unbound bilirubin is hampered by the lack of pathological correlates. ${ }^{16-18}$ Although, in theory at least, any amount of unbound bilirubin may jeopar- 
dise cerebral metabolism, it is not known how much unbound bilirubin must be present to produce bilirubin encephalopathy or whether there is a relation between the amount of unbound bilirubin and the severity of the cerebral insult. In this study we observed serum concentrations of apparent unbound bilirubin as high as $45 \mathrm{nmol} / \mathrm{l}$ without any detectable clinical signs of encephalopathy. Only one of the two babies who died during the study had a postmortem examination performed and he had no evidence of kernicterus; the maximal concentration of apparent unbound bilirubin in this baby was $16.4 \mathrm{nmol} / \mathrm{l}$.

Previous studies have indicated that neonates who received intermittent infusions of fat emulsions showed evidence of considerable fluctuations in free fatty acid:albumin molar ratios and also had very high ratios (in excess of 15). ${ }^{19}$ By comparison, this ratio did not fluctuate during continuous infusions of up to $2 \mathrm{~g} / \mathrm{kg}$ per day. Over $2 \mathrm{~g} / \mathrm{kg}$, however, the ratios reportedly averaged 10 to $16 .{ }^{19}$ This may be another argument in favour of continuous infusion of fat emulsions at a constant rate.

The observation that fat emulsions interfere with the spectrophotometric determination of serum bilirubin concentrations has been made previously. ${ }^{20}$ In that study, however, the presence of fat particles caused an overestimation in the concentration of conjugated bilirubin, not of total bilirubin. In the present study creaminess of a plasma sample was not judged in the same way in the nursery where the samples were obtained and in the laboratory where they were assayed. If a plasma sample was judged in the nursery to be excessively creamy-admittedly, a very subjective judgment-the study was stopped and the baby excluded from the study. If, on the other hand, an occasional total bilirubin concentration was not measurable or spuriously raised in the laboratory because of their assessment of excessive creaminess, that particular value was not reported but the baby was not excluded from the study.

This study was supported in part by grant HD 15967 from the National Institute of Child Health and Human Development, Bethesda, Maryland, and by KabiVitrum, Alameda, California.

\section{References}

${ }^{1}$ Brans YW. Parenteral nutrition of the very low birth weight neonate: a critical view. Clin Perinatol 1977;4:367-76.

${ }^{2}$ Hilliard JL, Shannon DL, Hunter MA, Brans YW. Plasma lipid levels in preterm neonates receiving parenteral fat emulsions. Arch Dis Child 1983;58:29-33.
${ }^{3}$ Brans YW. Erratum: values for plasma glycerol, FFA, and triglycerides. J Pediatr 1984;105:855.

${ }^{4}$ Brans YW, Dutton EB, Andrew DS, Menchaca EM, West DL. Fat emulsions tolerance in very low birthweight neonates: effect on diffusion of oxygen in the lungs and on blood $\mathrm{pH}$. Pediatrics 1986;78:79-84.

5 Dubowitz LMS, Dubowitz V, Goldberg C. Clinical assessment of gestational age in the newborn infant. J Pediatr 1970;77:1-10.

${ }^{6}$ Freeman MG, Graves WL, Thompson RL. Indigent negro and caucasian birthweight-gestational age tables. Pediatrics 1970;46:9-15.

7 Jacobsen J, Wennberg RP. Determination of unbound bilirubin in the serum of newborns. Clin Chem 1974;20:783-9.

${ }^{8}$ Doumas BT, Watson WA, Biggs HG. Albumin standards and the measurement of serum albumin with bromcresol green. Clin Chim Acta 1971;31:87-96.

9 Andrew G, Chan G, Schiff D. Lipid metabolism in the neonate. II. The effect of Intralipid on bilirubin binding in vitro and in vivo. J Pediatr 1976;88:279-84.

10) Thaler MM, Wennberg RP. Influence of intravenous nutrients on bilirubin transport II. Emulsified lipid solutions. Pediatr Res 1977;11:167-71.

1 Thiessen H, Jacobsen J, Brodersen R. Displacement of albumin-bound bilirubin by fatty acids. Acta Paediatr Scand 1972;61:285-8.

12 Jacobsen J, Thiessen H, Brodersen R. Effect of fatty acids on the binding of bilirubin to albumin. Biochem $J$ 1971;126:7.

${ }^{13}$ Brodersen R. Prevention of kernicterus, based on recent progress in bilirubin chemistry. Acta Paediatr Scand 1977;66:625-34.

${ }^{14}$ Starinsky R, Shafrir E. Displacement of albumin-bound bilirubin by free fatty acids. Implications for neonatal hyperbilirubinemia. Clin Chim Acta 1970;29:311-8.

15 Brodersen R. Free bilirubin in blood plasma of the newborn: effects of albumin, fatty acids, $\mathrm{pH}$, displacing drugs, and phototherapy. In: Stern L, Oh W, Friis-Hansen B, eds. Intensive care in the newborn. II. New York: Masson Publishing, 1978:331-45.

16 Ritter DA, Kenny JD, Norton HJ, Rudolph AJ. A prospective study of free bilirubin and other risk factors in the development of kernicterus in premature infants. Pediatrics 1982;69:260-6.

17 Cashore WA, Oh W. Unbound bilirubin and kernicterus in low-birth-weight infants. Pediatrics 1982;69:481-5.

${ }^{18}$ Maisels MJ. Clinical studies of the sequelae of hyperbilirubinemia. In: Levine RL, Maisels MJ, eds. Hyperbilirubinemia in the newborn. Report of the Eighty-fifth Ross Conference on Pediatric Research. Columbus, Ohio: Ross Laboratories, 1983:26-38.

19 Kao LC, Cheng MH, Warburton D. Triglycerides, free fatty acids, free fatty acids/albumin molar ratio, and cholesterol levels in serum of neonates receiving long-term lipid infusions: controlled trial of continuous and intermittent regimens. J Pediatr 1984;104:429-35.

20) Shennan AT, Cherian AG, Angel A, Bryan MH. The effect of Intralipid on the examination of serum bilirubin in the newborn infant. J Pediatr 1976;88:285-8.

Correspondence to Professor Y W Brans, Division of Neonatology, Westchester County Medical Center, Valhalla, New York 10595 United States of America.

Received 1 September 1986 